\title{
Method validation for mycotoxin analysis
}

\author{
Goto, T. \\ Faculty of Agriculture, Shinshu University \\ (8304 Minami Minowa-mura, Kamiina-gun, Nagano 399-4598, Japan)
}

\section{Summary}

Method validation is one of the key activities to ensure that analytical data and the method used to produce it have been verified before use. Harmonized collaborative validation (HCV) is the most prestigious form of method validation but it is not necessary to validate all methods by HCV. As each method has it's fit for purpose, the various forms of method validation have their fit for purpose. In the area of method validation, AOAC International leads the world, but there are currently many issues regarding the process of method validation itself. However, the use of validated methods is beginning and it is one of the keys for laboratory quality assurance.

Key words : method validation, harmonization, fit for purpose, LQA, HCV, AOAC

\section{Introduction}

Currently, quality assurance of data produced by analytical laboratories is an important issue. To ensure the quality of data, a number of efforts are taken as part of a laboratory quality assurance (LQA) plan. Among these, the use of validated methods is one of the key points. So, what is a validated method? What is method validation? How does one validate a method? In this paper, I would like to discuss chemical method validation, mainly based upon experience in mycotoxin method development and work with the AOAC International (AOAC) Official Methods of Analysis (OMA) program.

\section{Changes in analytical methodology}

For mycotoxin analysis, many methods have been developed and used for various mycotoxins in various commodities and also methods have been changed from time to time depending on available techniques or environmental requirements. Each method is specific for analyte (ex, aflatoxin, ochratoxin, patulin, or DON), matrix (ex, peanut, coffee, juice or wheat) and how it is conducted. So when a new analyte or matrix comes in, a new method has to be developed and validated. Or, if new techniques became available, a new method must be developed and validated. In Fig. 1, changes of the types of methods used for aflatoxin and ochratoxin analysis over time are shown. Until the 1980s, mainly TLC methods were used for the analysis of these mycotoxins probably because they have strong fluorescence and were easy to detect on the plate. However, more recently, HPLC methods have begun to dominate probably because of the requirement for improved accuracy. Also, extraction and clean up sample steps have changed. For extraction solvent, in 1970s and 1980s most methods used chlorinated solvents but today, the use of chlorinated solvents in newly developed methods is very rare. For clean up of extracted samples, liquid-liquid partitioning and open column 
chromatography were very popular but now one step solid phase extraction, multifunctional clean-up columns (MFC), and immuno-affinity clean-up column (IAC) have become more popular.

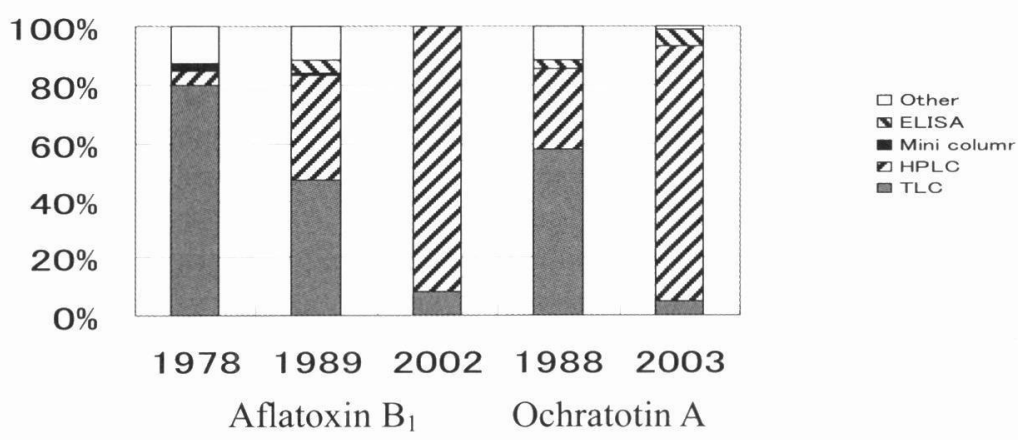

Fig. 1. Transition of mycotoxin analytical methods

Data from the 1970s and 1980s data were taken from the WHO mycotoxin check sample program and data from the 2000 s were taken from the FAPAS program.

\section{Methods and method validation}

Each analytical method was developed depending on a need for the method, and performance of the method must fit the purpose of the method. There are several requirements for methods that have to be considered during method development through method validation. For example, identification/selectivity for both analyte and matrix, limit of detection limit (LOD), limit of quantitation (LOQ), linearity, range, accuracy, repeatability, reproducibility, robustness, recovery, and uncertainty are key factors for any method. However, the relative importance of these factors may vary depending upon the purpose of the method. Also, depending upon the purpose of the method, the required performance of the method and required level of validation may vary. Therefore, the way of validating the method may depend upon the method. Some aspects of the method are mainly studied during the method development stage, and some of them are studied during the early stage of method validation such as during a single laboratory validation. Certain aspects, such as reproducibility, can be studied only through collaborative study.

At one time, AOAC tried to build a database named $e$-CAM. The concept of this data base was to classify methods depending on the levels of method validation. In the $e-C A M$, methods were classified into five groups: regulatory, harmonized collaborative validation (HCV), multi laboratory validation (MLV), single laboratory validation (SLV) and research unvalidated. Among these, regulatory was an exception because methods which were classified into this group were used for enforcement by regulatory agents and analysts needed to follow them regardless of the quality of the method. Research unvalidated methods were a pool for future validation. Methods for which validation was carried out following international protocols for single laboratory validation ${ }^{1)}$ or similar programs were classified as SLV. Methods validated by two or more numbers of laboratories were classified as MLV. In the AOAC, the peer verified method validation program (PVM) and the performance tested method validation program (PTM) are in this category. HCV is validation following internationally harmonized protocols ${ }^{2)}$ with many requirements. In the AOAC, OMA fits for this category and the OMA program ${ }^{3)}$ is internationally recognized as a most prestigious program for method validation. 
Validation is necessary for all analytical methods used but strategies for validation vary depending upon the purpose of the validation and the purpose of the method. For example, in the United States or European Union, HCV is the standard type of validation for methods used for regulatory purposes. In many countries, $\mathrm{HCV}$ is strongly recommended for regulatory methods. However methods without proper validation are occasionally used for regulatory purposes. $\mathrm{HCV}$ is an expensive and time consuming form of method validation, so it is not a suitable form of method validation for methods used only inside of one laboratory or with some types of commercial test kits. For these types of methods, MLV, SLV or a subset of these validation methods may be sufficient. This is fit for purpose of method validation. However, method validation and method verification are different. The purpose of method validation is to estimate performance of a method and the purpose of verification is to check the applicability of the method in the laboratory where the method is to be used.. Therefore, verification can be done only after the method has been validated on the analyte(s) and matrix(s).

\section{AOAC OMA and PTM method validation}

AOAC is a scientific organization founded in 1884 as a part of the U.S. Department of Agriculture. Since that time the improvement of the quality of analysis is the main interest of the society. Especially in method validation, AOAC leads the world.

AOAC's OMA first appeared as the Official and Provisional Methods of Analysis of the AOAC, in 1912. New versions of OMA are now published about every five years. The most recent issue of OMA (the 18th edition) was published in autumn of 2005 as a book and a Web version, and a 2006 revision appeared on the Web version only. In the OMA validation program, a large group of volunteers are involving in the validation process. The AOAC Official Method Board (OMB) leads the program. The OMB consists of the chair, vice chair, chairs of method committees and advisory committees, and a liaison from the AOAC Board of Directors. Each method committee (MC) is composed of a method committee chair (MCC), secretary, and 5 to 9 members. In addition to the MC members, General Referees (GRs) and Topic Advisors (TAs) work together to cover all aspects which the committee administers. Also, hen a method validation is conducted, a study director (SD) is appointed for each method and a group of volunteers work for the method as collaborators.

An outline of the OMA method validation process is provided in Fig. 2. In most cases the OMA process starts when the protocol for collaborative study is submitted to the AOAC from the organization that who wishes to validate the method. In most cases a person from that organization will be appointed the SD by the AOAC. First, the protocol and other assisting data for the method are reviewed by the GR and the safety and statistical advisors. After revision, if necessary, the MC reviews and the MCC approves the protocol. Once the collaborative study is finished, the SD writes and submits a manuscript to the GR. The GR and advisors reviews the study, which is then reviewed by the MC and two other OMB members. If acceptable, the method receives first action approval. AOAC has two types of MLV programs. One, for non-proprietary methods, is the PVM. The second, for proprietary methods or commercial kits, is the PTM. PVM is recognized as the preparatory step for OMA but is currently not very active. However, the interest for validation from commercial kit makers is very strong and the makers of many commercially sold kits, especially tests for microorganisms, desire authorization. The AOAC Research Institute (AOACRI), a subsidiary of the AOAC, operates a MLV program for commercial kits as a PTM. For PTM, AOACRI has a pool of scientists as reviewers of the method and a pool of laboratories to check the performance of the method as third parties.

The key to these programs is peer review. This peer review system is similar to the 
review of manuscripts which most scientific journals use. The difference is that the pool of reviewers have knowledge and/or experience not only with the analyte or matrix but also with the method validation itself.

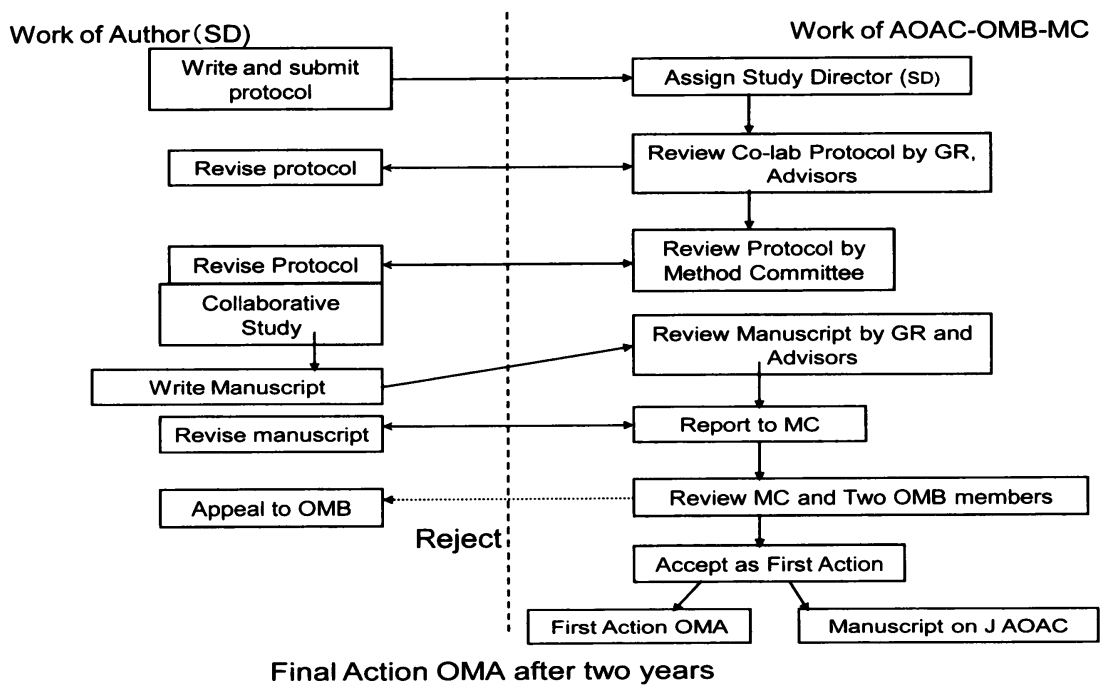

Fig. 2. AOAC-OMA validation Process

\section{Issues in method validation}

As previously mentioned, from method development to validation, there are many things those must be checked. Some of them have to be checked during method development and some of them can only be studied collaboratively. For example, identification/selectivity for the analyte and matrix(s) must be studied before the validation step because it is almost impossible to check all of the possible combinations of analyte and matrixes by collaborative study. The collaborative study must then be designed to use the expected matrix and analyte combinations in order to cover a wide range of matrices. Otherwise, the validated methods can only be used for a very limited number of matrices. Robustness of the method must also be tested before the collaborative study. This can be given in the protocol as allowances of the method. In some cases, LOD, LOQ, linearity and range are highly dependent upon the instruments used. In such cases, from the purpose of validation for the method, the requirements of performance of the method must be carefully checked. So, in the collaborative study protocol, it is a good idea to write requirements for the instrument by performance instead of specifying a particular instrument. If collaborative study protocol has specified only one instrument or one certain tool, the method can only use that instrument or tool. If someone would like to use a similar instrument or tool, they must re-validate the method. It is waste of resources.

Protocol is also very important for method validation, especially in the case of HCV. At the time of collaborative method validation, if the protocol does not provide the collaborators with enough information about how to perform the analysis, the study will become difficult and has a good chance to fail. The collaborators must follow the protocol, and the protocol must supply sufficient detail. In the protocol, information on how to perform the analysis as well as other information on samples, chemicals, tools, instruments, safety and the reporting of results must be included. Also, the protocols used for preparation of samples are an 
important for adequate review of the study. This document must not be shown to the collaborators, but must be to included in materials reviewed, along with other documents such as the detailed pre-collaborative study results.

The demand for validated methods is enormous and it has become impossible to validate all of the necessary methods through full collaborative study (HCV). For Asian counties, analytical methods with rice as the matrix are very important, but unfortunately there are few methods available. There are many methods available for wheat, barley or maize. How to extend the matrix applicability of a method is a major issue and the AOAC-OMB has agreed to use SLV for simple matrix extension without any changes to the method. Another issue is method modification. An example is the use of hyphenated instruments such as LC-MS, LC-MS/MS or GC-MS instead of TLC, HPLC or GC. Others include the use of new extraction solvents, or new clean-up procedures. There are so many things we must think about. In most case, minor modifications can be validated through SLV. However what kind of modification is minor? For this issue, there are no easy answers at the moment. The best answer at this time is through the decision by a third-party group of scientists who know the method, analyte, and matrix.

Another issue is the harmonization of method validation. As already mentioned, there are several ways to validate methods. Technically, most of requirements for method validation are similar from SLV to $\mathrm{HCV}$, therefore harmonized validation protocols can reduce the cost, and time, and improve the efficiency of the validation.

\section{Conclusion}

Method validation is one of the keys to ensure the quality of analytical data and many efforts must be made to incorporate this activity. For method validation, peer review is very important to ensure the properness of the validated method. However, method validation itself has it's fit for purpose, just as an analytical method has it's fit for purpose. With proper validation methods become trustworthy, but $\mathrm{HCV}$ is not the only mechanism for validating methods. It is very important to select the proper type of validation in order to validate good methods efficiently. The use of validated methods is a starting point of LQA activity. For LQA, the use of validated methods with proper verification, the use of internal quality control programs, and external quality assessment, such as the use of proficiency testing, and third party assessment of the activity are all important.

\section{References}

1) Thompson, M., Ellison, S.L.R. and Wood, R.: Pure Appl. Chem., 74, 835-855 (2002)

2) J.AOAC Int, 78, 143A-160A(1995)

3) http://www.aoac.org/vmeth/oma_program.htm 